\title{
The Effect of Anti-anxiety Medications on the Clinical Symptoms of Anal Fissure: A Randomized Clinical Trial
}

\author{
Ali Mirsadeghi' ${ }^{1}$ Narges Novin ${ }^{2}$, Seyed Mohammad Ali Raisolsadat ${ }^{3,}$, , Maryam Javanbakht ${ }^{4}$ and Tooraj Zandbaf ${ }^{1}$ \\ ${ }^{1}$ Assistant professor of General Surgery, Faculty of Medicine, Islamic Azad University, Mashhad Branch, Mashhad, Iran \\ ${ }^{2}$ General Practitioner, Faculty of Medicine, Islamic Azad University, Mashhad Branch, Mashhad, Iran \\ ${ }^{3}$ Assistant Professor of Pediatric Surgery, Faculty of Medicine, Islamic Azad University, Mashhad Branch, Mashhad, Iran \\ ${ }^{4}$ Assistant Professor of Psychiatry, Faculty of Medicine, Islamic Azad University, Mashhad Branch, Mashhad, Iran
}

* Corresponding author: Seyed Mohammad Ali Raisolsadat, Faculty of Medicine, Islamic Azad University, Mashhad Branch, Mashhad, Iran. Tel: +989151158808; Email: Raeisolsadat1458@mshdiau.ac.ir

Received 2020 June 21; Revised 2020 October 16; Accepted 2020 November 13.

\begin{abstract}
Background: Fissure is a common chronic anorectal disease that causes symptoms such as pain, burning, bleeding, and psychiatric problems. One of the psychiatric complications is anxiety, which might be an etiologic factor or secondary to the disease.

Objectives: The present study aimed to investigate the effect of anti-anxiety medications on the clinical symptoms of patients with anal fissures.

Methods: A total of 160 patients with anal fissure were randomly divided into two groups (80 patients treated with anti-anxiety medications along with standard anal fissure treatment and 80 patients with standard anal fissure treatment alone) after obtaining patient consent and demographic data. Then, the symptoms have recorded after treatment.

Results: The results of our study indicated that the administration of anti-anxiety medications in patients with anal fissures relieved pain and anxiety scores among all patients. The anti-anxiety medications were more effective on patients younger than 40 years, men with less than two weeks duration of illness, and baseline anxiety greater than 26 .

Conclusion: A high prevalence of anal fissures affects the quality of life of patients. Besides the lack of effective treatment that shortens the pain and recovers patients faster, anti-anxiety medications such as benzodiazepines can use along with standard anal fissure treatment to reduce pain and anxiety in patients with acute anal fissure.

Keywords: Anal fissure, Anti-anxiety medications, Benzodiazepines
\end{abstract}

\section{Background}

Fissure is a common anorectal chronic disease associated with pain and bleeding during defecation $(1,2)$. The disease presents with rupture of the anoderm area due to trauma from passing hard stools or prolonged diarrhea $(3,4)$. It affects about $1 \%$ of the world population, with the highest prevalence in women over 56. Severe pain and bleeding during defecation are associated with internal spasms of the internal anal sphincter. Also, the reduction of blood flow due to fissures reduces the speed of recovery. While many believe that the disease can spontaneously heal, studies have shown that patients who still have symptoms after 4 to 5 weeks need medical intervention (5). An anal fissure can gradually reduce the quality of life of patients for various reasons, including depression, recurrent bleeding, and disability (6). Pain and chronic disease symptoms as well as the treatment process and its high costs cause significant anxiety in these patients (7).

\section{Objectives}

The disease has a relatively high prevalence globally and its effects (especially anxiety) have been proven on the quality of life of patients.
Unfortunately, to the best of our knowledge, no relevant study was found in the conducted searches. Therefore, the present study aimed to investigate the effect of anti-anxiety medications on the clinical symptoms of patients with anal fissures.

\section{Methods}

\subsection{Trial design}

This study is a prospective double-blinded randomized clinical trial and includes all patients with anal fissures referred to 22 Bahman Hospital, Mashhad, Iran, between November 2019 and December 2020. The present study has been approved by the Ethics Committee of Islamic Azad University, Mashhad Branch, Mashhad, Iran (IR.IAU.MSHD.REC.1398.150) on 2019-10-30 and was conducted according to the principles of the Declaration of Helsinki and good clinical practice guidelines. The work has been reported following Consolidated Standards of Reporting Trials (CONSORT) Guidelines.

\subsection{Participants}

Inclusion criteria included patients with an acute anal fissure, aged between 20 - 62 years, who had no evidence of a chronic fissure such as sentinel tag in 
clinical examination and any contraindication for the use of anti-anxiety medications. The patients completed the informed consent sheet, checklist, and relevant questionnaires during the interview. Exclusion criteria were individuals with evidence of chronic anal fissure on clinical examination, who were reluctant to participate in the study, patients who could not follow up, those with abnormal levels of anxiety according to the Spielberger questionnaire, and individuals with a history of psychiatric illness or being treated with psychiatric medications.

\subsection{Intervention}

In this study, 160 patients with anal fissures were included who were eligible for inclusion criteria with no exclusion criteria. According to the previous Spielberger questionnaire, a checklist was prepared for each patient, including personal information, age, gender, duration of disease, and levels of pain, bleeding, and anxiety. This study was double-blinded, therefore the researchers asked a trusted third party to provide 3200 empty capsules. He should prepare 800 blue and 800 red capsules, containing 0.5 and $0.25 \mathrm{mg}$ of alprazolam, respectively. He had to make a package with a specific code for each of these capsules. Also, he was required to prepare 800 blue and 800 red capsules containing starch; then, he had to prepare a package containing both types of capsules and assign a specific code to them and keep the relevant information. In this study, patients were randomly assigned to two separate groups A and B, based on a table of random numbers. After obtaining informed consent, each patient completed a checklist, the visual analog scale (VAS) of pain, and Spielberger's questionnaire. We simultaneously prescribed two coded capsules with standard treatment of fissure according to the table of random numbers (two capsules per day; one red capsule every morning and one blue capsule at night).

\subsection{Outcomes}

After 12 days, we again checked and recorded the severity of anal bleeding, the amount of pain (visual analog scale of pain questionnaire), and anxiety level (Spielberger's questionnaire) in both groups of patients.

\subsection{Instruments to measure outcomes}

a) The visual analog scale (VAS) is a frequently used pain measurement technique. The patients were asked to rate their intensity of pain along a $100 \mathrm{~mm}$ horizontal line (most commonly), and then the rate was measured from the left edge (=VAS score). According to Myles et al., the VAS score is a linear scale in postoperative patients with acute mild-to-moderate pain. Variations in the VAS score indicate a change in the magnitude of the pain experience. As a result, VAS may now be used to assess variations in potency and efficacy in comparative analgesic trials. (8) b) The State-Trait Anxiety Inventory (STAI) was created by Spielberger (1812), who introduced the first form of the adjective mood scales, and in 1893 presented its revised version (9). STAI consists of 40 self-reported items that the first 20 measure the state, and the rest measure the anxiety trait. This scale has considerable credibility and reliability. The state scales were used in this study. This questionnaire has twenty possibilities ranging from 1-4 (1= very low, $2=$ low, $3=$ high, and $4=$ very high). A minimum of 20 to a maximum of 80 marks is considered for items 1-20 of the Trait and State Questionnaire. Therefore, the score of each subject will be between 20 to 80 in each form. According to Gholami's study (2017), the reliability coefficient of this scale was reported to be 0.93 for the state scale and 0.90 for the trait scale in Iran. Also, assuming appropriate validity of the Cattle Anxiety Inventory, the frequency correlation between the STAI questionnaire and Cattle Anxiety Inventory is 0.93 which is positive and significant (9). Marteau and Bekker (1992) and Spielberger and Garoush (1970) also reported good validity and reliability for the tool $(10,11)$.

\subsection{Sample size calculation}

We use Cochran's sampling formula for finding a statistical sample size (12). Here, the minimum sample size was 56 patients for $95 \%$ confidence level, $5 \%$ margin of error, and the size of our research population (168). However, we worked with 80 patients in each case and control group.

\subsection{Randomization}

In this study, patients were randomly assigned to two separate groups A and B based on a table of random numbers.

\subsection{Statistical method}

In data analysis, the normality of data was investigated using the Kolmogorov-Smirnov test with Lilliefors correction. Appropriate parametric methods such as the Student t-test were used to confirm normality, and if it was not normal, we used the Mann-Whitney U test. Pearson's Chi-Squared test was used to analyze nominal scale data, and Fisher's exact test was performed in cases where more than $20 \%$ of the expected frequencies of the tables were less than 5 (Cochran). SPSS software (version 21) was used for data analysis. A p-value less than 0.05 is statistically significant.

\section{Results}

\subsection{Participants in the study and baseline characteristics}

Figure 1 shows the CONSORT flow diagram of the trial. A total of 168 patients with anal fissures who were eligible for the inclusion criteria were included and 160 patients were finally analyzed between 


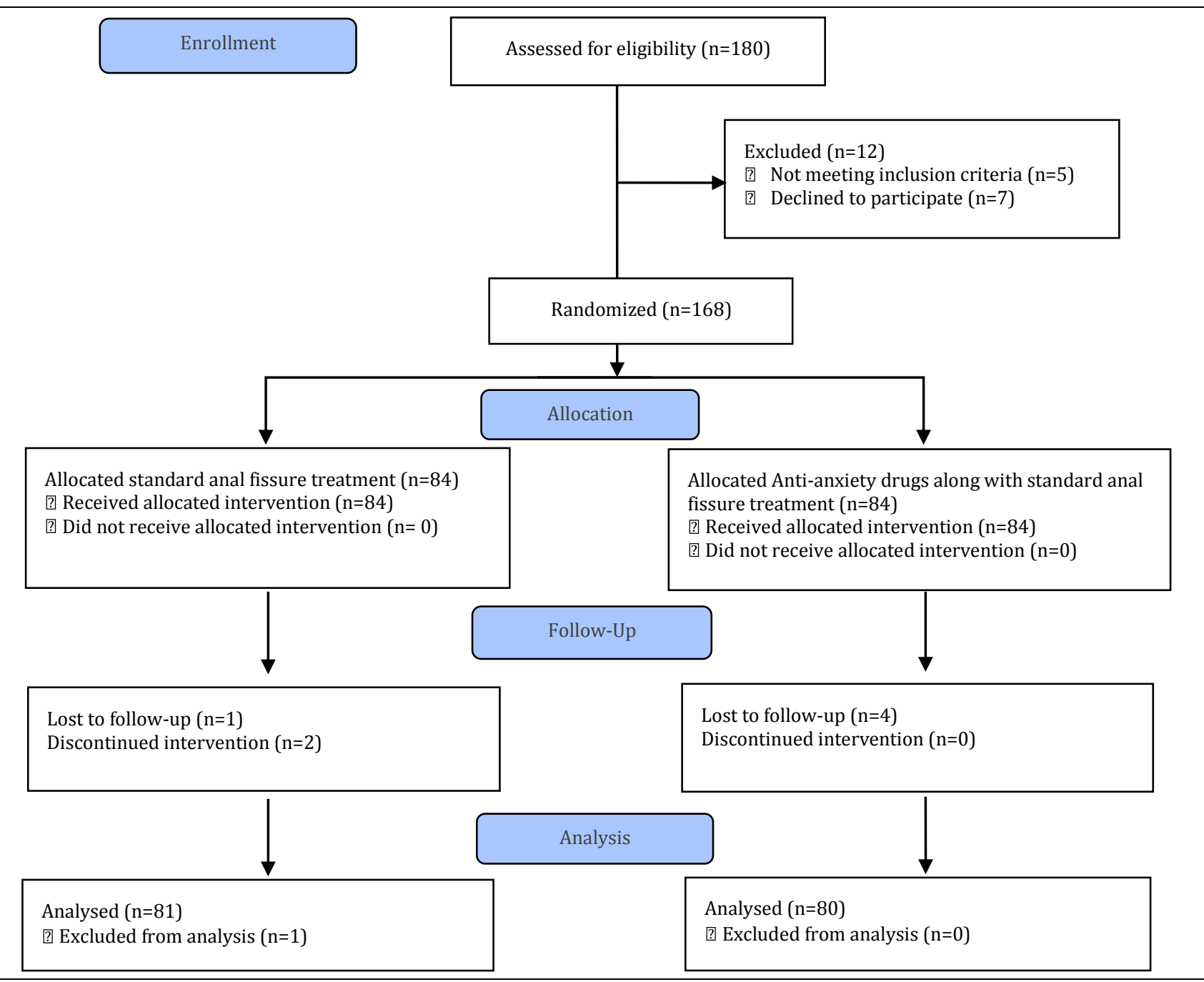

Figure 1. CONSORT diageram of trial

November 2019 and December 2020.

Eighty patients were treated with anti-anxiety medications in addition to the standard treatment of fissures and 80 patients were only treated with standard treatment), of which 93 (58.1\%) were men and $67(41.9 \%)$ were women. Also, the mean age of the subjects was $33.32 \pm 8(20-50$ years old $)$. In the present study, age $(P=0.18)$, gender $(P=0.631)$, and the duration of disease $(\mathrm{P}=0.548)$ were not significantly different between the two groups. Also, the mean pain was not statistically significant in patients of the two groups according to the visual analog scale, before the therapeutic intervention ( $P$ $\geq 0.05$ ). However, it was found that the mean pain was significantly lower in the intervention group after treatment $(3.9$ vs. $4.5, \quad \mathrm{P}=0.018)$. This difference remained significant in patients under 40 years of age, men, duration of disease less than two weeks, and baseline anxiety over 26 . Also, according to the Wilcoxon test, the mean pain significantly decreased in both groups based on the visual analog scale $(\mathrm{P} \leq 0.001)$ (Table 1$)$.
Our findings also showed the number of pads used in the days before and after the treatment intervention. The mean bleeding was not statistically significant in patients of the two groups $(P \geq 0.05)$, which was still not significant by grouping patients based on age, gender, and duration of infection. However, the Wilcoxon test showed that the average bleeding was significantly reduced in both groups based on the number of pads used per day $(\mathrm{P} \leq 0.05)$ (Table 1$)$.

Finally, we realized that the mean anxiety was not statistically significant in patients in the two groups before the medical intervention $(\mathrm{P} \geq 0.05)$. However, after treatment, it became clear that the mean level of anxiety in the intervention group that received the anti-anxiety medications was significantly lower than that of the control group (21.7 vs 26.38) ( $\mathrm{P} \leq 0.001)$ and remained significant in all ages, gender, and disease groups. Also, the average anxiety significantly decreased in the intervention group based on the Wilcoxon test $(\mathrm{P} \leq 0.001)$ (Table 1). 


\begin{tabular}{lcccc}
\hline \multicolumn{4}{l}{ Table 1. Mean and standard deviation of the variables studied in patients. } & \\
\hline Variable & Intervention (80) & Control (80) & P-value \\
\hline Age (year) & $34.88 \pm 7.31$ & $33.44 \pm 9.25$ & 0.18 \\
Gender (male) & $48(\% 60)$ & $45(\% 56.2)$ & 0.631 \\
Duration of disease (week) & $3.71 \pm 3$ & $3.34 \pm 2.25$ & 0.548 \\
Pain (based on VAS) & Before intervention & $7.66 \pm 1.43$ & $7.59 \pm 1.42$ & 0.8 \\
& After intervention & $3.9 \pm 1.19$ & $4.51 \pm 1.72$ & 0.018 \\
Bleeding (number of pads in a day) & Before intervention & $1.45 \pm 0.61$ & $1.5 \pm 0.74$ & 0.838 \\
& After intervention & $1.34 \pm 0.55$ & $1.34 \pm 0.57$ & 0.909 \\
Anxiety & Before intervention & $27.28 \pm 2.32$ & $26.69 \pm 2.76$ & 0.177 \\
\hline & After intervention & $21.7 \pm 1.26$ & $26.42 \pm 2.65$ & 0.001 \\
\hline
\end{tabular}

\section{Discussion}

According to our findings, the mean pain, anxiety, and severity of bleeding were not statistically significant in patients of both groups before interventional treatment. However, it was found that the mean pain was significantly lower in the intervention group that received the anti-anxiety medications than that of the control group after treatment (3.9 vs. 4.5). This difference remained significant in patients under 40 years old, men, individuals with a disease duration of fewer than two weeks, and those with baseline anxiety over 26. However, the mean bleeding was not significantly different in both groups based on the number of pads used in the days before and after the treatment intervention. We found that the mean anxiety was significantly lower in the intervention group who received the anti-anxiety medications than that of the control group after treatment (21.7 vs. 26.38), which remained significant in all ages, gender, and disease duration groups.

Raisolsadat et al. (2008) conducted a prospective evidence-based case study on 83 patients with anal fissures based on the diagnosis of a general surgeon and 83 normal individuals as a control group. The Spielberger State-Trait Anxiety Inventory and the demographic information questionnaire designed by the researcher were completed by individuals and the review of the researcher. The results were analyzed using logistic regression and the chi-squared test. There were 19 men and 64 women in each group. The groups were similar in terms of age, gender, education level, and marriage. Trait anxiety showed significant differences between both genders in the experimental and control groups (female $\mathrm{P}=0.0085$, male $\mathrm{P}=$ 0.0206). No difference was observed in state anxiety. There was a significant difference between the women in the two groups at higher levels of anxiety in terms of the chi-squared test (state anxiety $\mathrm{P}=0.15$ and trait anxiety $\mathrm{P}=0.010$ ). There was a significant correlation between trait anxiety and anal fissure in both genders. This relationship was not observed between the two genders in the case of state anxiety, and there was an association between the severity of anxiety and anal fissures at least in females. In this study, anti-anxiety therapy was initiated for some patients, and a significant reduction was observed in the severity of anxiety (7). In our study, the therapeutic goal was not to assess anxiety because all patients entered the study with a score of 20 to 31 (mild anxiety). However, consistent with the above study, treatment significantly reduced the mean score of anxiety in the intervention group which remained significant in all groups of age, gender, and duration of illness.

Ozden Arisoy et al. (2016) examined 30 patients with chronic anal fissure and 20 healthy individuals in a cross-sectional study. In this regard, the level of anxiety and depression of the participants was investigated by the SF-36 questionnaire. Anxiety and depression scores in the group with chronic anal fissures were significantly higher than that of the control group $(\mathrm{P} \leq 0.001)$. Primary and secondary stress were observed in $36 \%$ and $56 \%$ of patients, respectively. Pain $(96.7 \%$ had pain) and bleeding (83.3\% had bleeding) had a severe negative effect on patients and caused physical pain and mental limitations. The present study approved that chronic fissure disease increases depression and anxiety and reduces the quality of life of the patients. Stress has also been suggested as a cause of chronic anal fissure disease, which exacerbates it (12). Although the therapeutic goal of our study was not examining anxiety, because all patients entered the study with a score of 22 to 31 (mild anxiety), patients in the intervention group significantly responded to antianxiety treatment, which proves the increased level of stress in patients with fissures. This stress is related to their illness so that proper treatment can improve their stress condition.

Hang et al. (2017) conducted a study to investigate the effect of diazepam on patients with anal fissures. The patients were treated with diazepam between January 2013 and February 2015 at a comprehensive outpatient center. All 18 patients who received diazepam positively responded to pain, while $47.4 \%$ experienced a complete response, with a numerical scale score of 0 (NRS) (0-10). Also, $94 \%$ of patients positively responded to anal bleeding with an anal bleeding score (ABS) of 2 (9-2). Finally, it was concluded that diazepam significantly reduced pain and bleeding in the anal fissure (13). The findings of the study on wound healing are similar to those of our study, however, the effect of anti-anxiety medications on bleeding is inconsistent with our study findings. This discrepancy may be due to differences in sample size, 
patient demographic indicators, inclusion and exclusion criteria, prescription medications and dosage, and variable measurements.

Burston et al. discovered that anxiety was associated with increased pain responses in chronic pain states. The degree to which anxiety causes chronic pain or vice versa is a critical issue with implications for analgesic care. The level of pain and anxiety is measured in individuals with (130) or without (100) painful knee osteoarthritis. Higher levels of anxiety were associated with patients with osteoarthritis who had slightly lower levels of pain detection at locations close to $(P, 0.01)$ and far from $(P, 0.05)$ the painful knee. These findings showed that pain causes anxiety or vice versa, which is similar to our research, showing that anti-anxiety medications reduced the symptoms (pain) of anal fissures (14).

Westbrook et al. conducted a study on 100 patients who daily received $1.5 \mathrm{mg}$ of alprazolam to evaluate the administration of benzodiazepines in patients with chronic pain. No change was observed in medications or therapeutic interventions. Sixteen patients were excluded, and one oncologic case died at the follow-up. Of the 83 patients evaluated in 12 weeks, 61 patients (73.5\%) showed improvement, and only five patients discontinued the medications due to side effects. The mean scores of all patients showed a significant decrease in pain from 3.6 to 2.2 on the visual analog scale (15). Although the type of patients studied was different from our cases, the findings were consistent with our study and showed that controlling anxiety reduces pain in patients with chronic pain.

\section{Conclusion}

The results of our study indicated that prescribing anti-anxiety medications reduced pain and anxiety in patients with anal fissures (especially in individuals under 42 years, men with a disease duration of fewer than two weeks, and baseline anxiety score over 26 ). Anti-anxiety medications such as benzodiazepines can be used along with standard anal fissure treatment to reduce pain and anxiety in patients with acute anal fissure due to the high prevalence of anal fissure and a decrease in quality of life in patients, and lack of effective treatment that reduces the pain and recover patients faster.

\section{Footnotes}

Ethical Approval: The present study has been approved by the Ethics Committee of Islamic Azad University, Mashhad Branch, Mashhad, Iran (IR.IAU.MSHD.REC.1398.150) on 2019-10-30 and was conducted according to the principles of the Declaration of Helsinki and good clinical practice guidelines.

Funding: The authors did not receive financial support from any organization for the submitted article.

Credit Author Contribution Statement: AM: Data curation, Writing- Preparing Original draft; NN: Investigation, Formal analysis; SMAR: Conceptualization, Methodology; MJ: Methodology, Investigation; TZ: Writing- reviewing and editing.

Conflict of Interest: The authors declare that there is no conflict of interest regarding the publication of this paper.

\section{References}

1. Beaty J, Shashidharan M. Anal Fissure. Clin Colon Rectal Surg. 2016;29(01):030-7. doi: 10.1055/s-0035-1570390

2. Emile SH, Elgendy H, Elfeki H, Magdy A, Abdelmawla AA Abdelnaby $\mathrm{M}$, et al. Does the duration of symptoms of anal fissure impact its response to conservative treatment? A prospective cohort study. Int J Surg. 2017;44:64-70.doi: 10.1016/j.ijsu.2017.06.044

3. Zaghiyan K, Fleshner P. Anal Fissure. Clin Colon Rectal Surg . 2011;24(01):022-30. doi:10.1055/s-0031-1272820

4. Schlichtemeier S, Engel A. Anal fissure. Aust Prescr. 2016;39(1):14-7.doi:10.18773/austprescr.2016.007:

5. Mapel DW, Schum M, Von Worley A. The epidemiology and treatment of anal fissures in a population-based cohort. BMC Gastroenterol. 2014;14(1):2-8.

6. Griffin N, Acheson AG, Tung P, Sheard C, Glazebrook C, Scholefield JH, et al. Quality of life in patients with chronic anal fissure. Color Dis. 2004;6(1):39-44.doi: 10.1111/j.14631318.2004.00576.x

7. M.A. Raisolsadat, H.R Arshadi, M. Javanbakht BJE. On the relationship between anxiety and anal fissure Mohammad. J Fundam Ment Heal. 2009;1:69-74.

8. Myles PS. The Pain Visual Analog Scale: Linear or Nonlinear?. Anesthesiology. 2004;100(3):744.doi: : 10.1097/00000542200403000-00042

9. Gholami Booreng F, Mahram B, Kareshki H. Construction and Validation of a Scale of Research Anxiety for Students. Iran J Psychiatry Clin Psychol. 2017;23(1):78-93.doi: 10.18869/nirp.ijpcp.23.1.78

10. Marteau TM, Bekker H. The development of a sixitem short form of the state scale of the Spielberger State-Trait Anxiety Inventory (STAI). Br J Clin Psychol. 1992;31(3):301-6.doi: 10.1111/j.2044-8260.1992.tb00997.x

11. SPIELBERGER, D. C. Manual for the State-trait Anxietry, Inventory. Consult Psychol.2021

12. Arısoy Ö, Şengül N, Çakir A. Stress and psychopathology and its impact on quality of life in chronic anal fissure (CAF) patients. Int J Colorectal Dis. 2017;32(6):921-4.doi: 10.1007/s00384016-2732-1

13. Hang MTH, Smith BE, Keck C, Keshavarzian A, Sedghi S. Increasing efficacy and reducing side effects in treatment of chronic anal fissures. Med (United States). 2017;96(20).doi: 10.1097/MD.0000000000006853

14. Burston JJ, Valdes AM, Woodhams SG, Mapp PI, Stocks J, Watson DJG, et al. The impact of anxiety on chronic musculoskeletal pain and the role of astrocyte activation. Pain. 2019;160(3):658-69.doi: 10.1097/j.pain.0000000000001445

15. Westbrook L, Cicala RS, Wright H. Effectiveness of alprazolam in the treatment of chronic pain: Results of a preliminary study. Vol. 6, Clinical Journal of Pain. 1990:32-6.doi: 10.1097/00002508-199003000-00006 